Proceedings

\title{
Do neighbourhood and structural features affect health literacy in Polish adolescnts?
}

\author{
Dorota Kleszczewska *, Joanna Mazur, Anna Dzielska
}

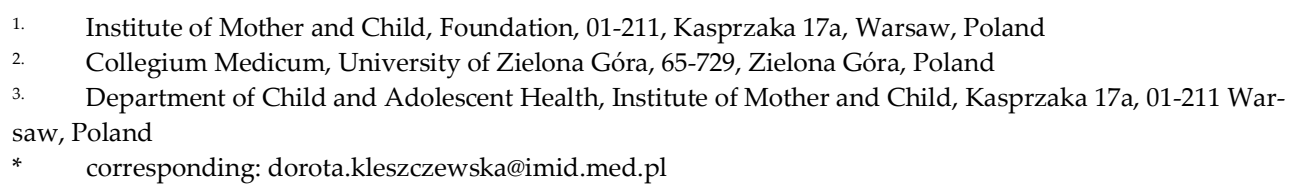

Abstract: BACKGROUND: Developing programmes to improve adolescents' health competences defined as health literacy (HL) seems to be crucial for their current and future health. OBJECTIVE: To understand if living in regions with low deprivation and high social capital increases HL in adolescence. MATERIAL AND METHODS: The survey was conducted in the 2018 as part of the HBSC study involving 5648 Polish students aged 13-17, 47,3\% boys. Adolescents' self-reported HL was measured with a brief 10-item Health Literacy for School-Aged Children (HLSAC) instrument (IJERPH, Paakkari et al. 2020). HLSAC was developed as a set of competencies to promote and sustain health (5 dimensions of HL covered, index ranged 10-40, mean $30.64 \pm 4.55$ ). As independent variables: gender, age group, neighbourhood social capital scale (as social feature), subjective assessment of neighbourhood affluence (as structural feature), family affluence (FAS) were used. Comparison of mean HL indexes by nonparametric Kruskal-Wallis test and General Linear Model (GLM) with 2-way interactions were applied.RESULTS: The average HL index increases from 29.94 to 31.85 along with the neighbourhood social capital $(\mathrm{p}<0.001)$, as well as the neighbourhood affluence ( from 29.26 to 31.43; $\mathrm{p}<0.001$ ). An significant interaction between FAS and the neighborhood affluence $(\mathrm{p}=0.004)$ means the accumulation of risk and protective factors (HL index ranges from 26.97 to 31,64 ) and a much stronger protective effect of the neighborhood features in the poor families as compared to average and high FAS groups. CONCLUSION: Environmental features should be analyzed in research on social determinants of HL and in planning targeted interventions.

Keywords: health literacy, adolescents, neighbourhood

Publisher's Note: MDPI stays neutral with regard to jurisdictional claims in published maps and institutional affiliations.

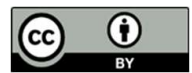

Copyright: (c) 2020 by the authors. Submitted for possible open access publication under the terms and conditions of the Creative Commons Attribution (CC BY) license (http://creativecommons.org/licenses /by/4.0/). 